\title{
Motion as a phenotype: the use of live-cell imaging and machine visual screening to characterize transcription-dependent chromosome dynamics
} David A Drubin ${ }^{1}$, Arman M Garakani² and Pamela A Silver*1

Address: ${ }^{1}$ Department of Systems Biology, Harvard Medical School and Department of Cancer Biology, The Dana-Farber Cancer Institute, Boston, MA, USA and ${ }^{2}$ Reify Corporation, Cambridge, MA, USA

Email: David A Drubin - david_drubin@hms.harvard.edu; Arman M Garakani - arman@reifycorp.com;

Pamela A Silver* - pamela_silver@hms.harvard.edu

* Corresponding author

Published: 24 April 2006

BMC Cell Biology 2006, 7:19 doi:10.1186/147/-2121-7-19
Received: 23 December 2005

Accepted: 24 April 2006

This article is available from: http://www.biomedcentral.com/I47/-2/2I/7//9

(C) 2006 Drubin et al; licensee BioMed Central Ltd.

This is an Open Access article distributed under the terms of the Creative Commons Attribution License (http://creativecommons.org/licenses/by/2.0), which permits unrestricted use, distribution, and reproduction in any medium, provided the original work is properly cited.

\begin{abstract}
Background: Gene transcriptional activity is well correlated with intra-nuclear position, especially relative to the nuclear periphery, which is a region classically associated with gene silencing. Recently however, actively transcribed genes have also been found localized to the nuclear periphery in the yeast Saccharomyces cerevisiae. When genes are activated, they become associated with the nuclear pore complex (NPC) at the nuclear envelope. Furthermore, chromosomes are not static structures, but exhibit constrained diffusion in real-time, live-cell studies of particular loci. The relationship of chromosome motion with transcriptional activation and active-gene recruitment to the nuclear periphery has not yet been investigated.
\end{abstract}

Results: We have generated a yeast strain that enables us to observe the motion of the galactoseinducible GAL gene locus relative to the nuclear periphery in real-time under transcriptionally active and repressed conditions. Using segmented geometric particle tracking, we show that the repressed GAL locus undergoes constrained diffusive movement, and that transcriptional induction with galactose is associated with an enrichment in cells with GAL loci that are both associated with the nuclear periphery and much more constrained in their movement. Furthermore, we report that the mRNA export factor Sac3 is involved in this galactose-induced enrichment of GAL loci at the nuclear periphery. In parallel, using a novel machine visual screening technique, we find that the motion of constrained GAL loci correlates with the motion of the cognate nuclei in galactoseinduced cells.

Conclusion: Transcriptional activation of the GAL genes is associated with their tethering and motion constraint at the nuclear periphery. We describe a model of gene recruitment to the nuclear periphery involving gene diffusion and the mRNA export factor Sac3 that can be used as a framework for further experimentation. In addition, we applied to the analysis of chromosome motion a machine visual screening approach that used unbiased visual data rather than segmented geometric data. This novel analytical approach will allow for high-throughput study of processes that can be monitored via alterations in chromosome motion and connectivity with the nuclear periphery. 


\section{Background}

The separation of transcription and mRNA processing in the nucleus from cytoplasmic translation affords the eukaryotic cell an added level of regulatory complexity for gene expression. Additionally, the nucleus provides an architectural framework in which the chromosomes are non-randomly organized into chromosome territories, although the position of chromosomes within the nucleus varies between cell types and among populations of cells (for review, see ref. [1]). This variation in chromosome positioning is thought to reflect certain properties of the chromosome, such as size and transcriptional activity $[2,3]$.

The relationship of transcriptional activity with the intranuclear positioning of genes has been well documented, particularly relative to the periphery of the nucleus (for reviews, see ref. [4-6]). Localization at the nuclear periphery is traditionally a hallmark of gene silencing. The cystic fibrosis transmembrane regulator (CFTR) gene, when silent, is associated with the nuclear periphery, but is more interior when expressed [7]. In the budding yeast Saccharomyces cerevisiae, silenced telomeres are localized to the nuclear periphery via the nuclear pore complex (NPC) [8-11], and repression of the silent mating type loci is associated with NPC components and the nuclear periphery $[8,9,12]$.

However, recent studies have suggested that the association with the nuclear periphery is not exclusively for gene silencing. One such study in S. cerevisiae demonstrated the activation of a reporter gene by NPC components and nuclear transport machinery via a boundary activity that isolates the gene from silent chromatin [13]. We have since demonstrated through a yeast genome-wide localization analysis that components of the NPC and transport machinery are associated with a subset of actively transcribed genes, as well as the expected silenced genes [14]. Furthermore, we have shown inducible genes involved in galactose metabolism, specifically the GAL1, 7, and 10 genes (GAL locus), shift from the nuclear interior to the NPC upon growth in galactose [14]. We also observed recruitment of genes to the NPC from thirteen different chromosomes that are activated in response to the yeast pheromone alpha factor [15]. Recruitment of the INO1 gene to the nuclear periphery upon transcriptional induction [16], and the association of several NPC components with transcriptional activation [17] both provide further evidence that the nuclear periphery is a complex region of transcriptional regulation.

Although there is a defined shift in position of certain activated genes from the nuclear interior to the NPC, the mechanism and functional significance of this phenomenon remain unclear. Chromosomes exhibit a constrained diffusive motion [18-20], which may be a means of bringing genes into association with the nuclear periphery. Transcription itself, as indicated by the RNA dependency for association of alpha factor-induced genes with the NPC [15], may be involved. Furthermore, specific connecting factors may include components of the mRNA export machinery that also exhibit associations with the transcriptional machinery.

In the study presented here, we report that $G A L$ locus motion becomes tightly constrained at the nuclear periphery upon transcriptional induction. From this and the observation of transient "sampling" of the nuclear periphery by the GAL locus, we propose a model of active-gene recruitment to the nuclear periphery. Furthermore, we present the use of real-time chromosome motion in live cells coupled with machine visual screening as a reporter system for gene localization at the nuclear periphery, and propose its application to other studies of this type.

\section{Results}

Visualizing GAL locus position and movement in living cells To study the chromosome dynamics associated with transcription, we tagged the GAL locus in a haploid strain of Saccharomyces cerevisiae by integrating an array containing 256 lac operator (lacO) sites into the intergenic region between the GAL7 and GAL10 genes, and co-expressing a green fluorescent protein-lac repressor fusion (GFP-lacI); (Figure 1a) [18-22]. The site of integration preserves the promoter elements of GAL7 and the 3' elements of GAL10, such that the strain exhibits normal regulation of the $G A L$ system (data not shown). In addition to marking the GAL locus, we also fused two nuclear pore proteins, Nup49 and Nup60, with a yeast codon-optimized DsRed variant, tdimer2 $[23,24]$ in order to mark the nuclear periphery [18].

To determine the ability of our live-cell fluorescence system to correctly report the position of the GAL locus, we grew cells in YP-raffinose and split the culture into YPgalactose (induction) and YP-glucose (repression) media for 3-hours. Cultures were then collected and placed on $2 \%$ agarose pads on glass slides for imaging. Image sequences (captured every $5 \mathrm{~s}$, for $5 \mathrm{~min}$ ) were generated from cells in which the GAL locus spot was visible and used to score whether the GAL locus was at the periphery for the entire time of the image sequence or not (Figure $1 \mathrm{~b}, \mathrm{c})$. This analysis was repeated for four separate galactose and glucose treatments, and we observed a similar trend as previously reported for GAL position [14]; about $50 \%$ of cells had the GAL locus at the periphery upon transcriptional induction, and only $28 \%$ in repressed conditions (Figure 1d). 
a

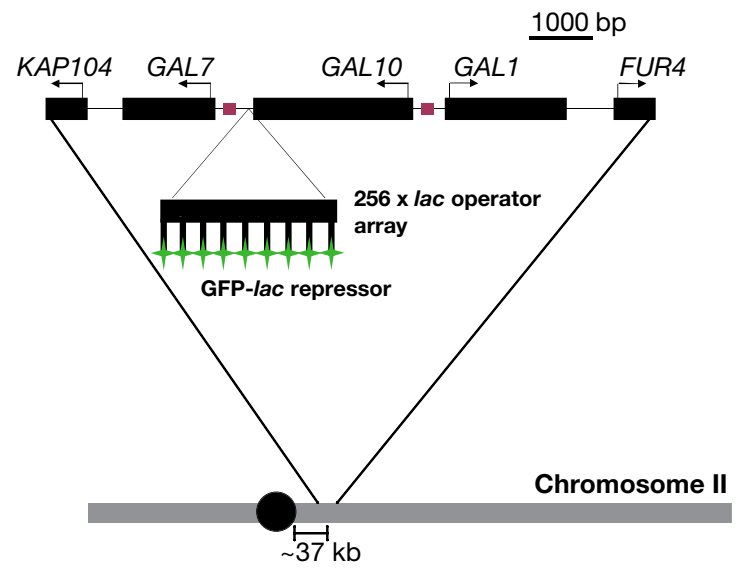

c

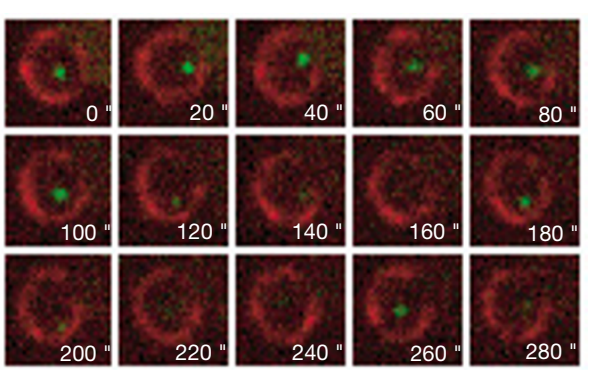

b
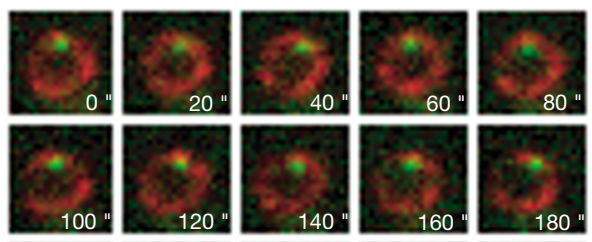

rastar
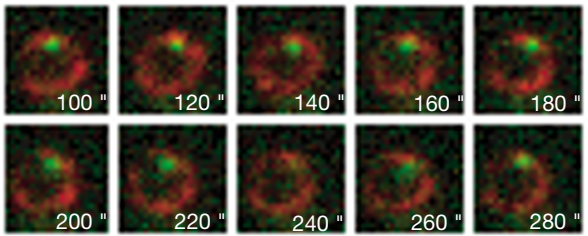

d

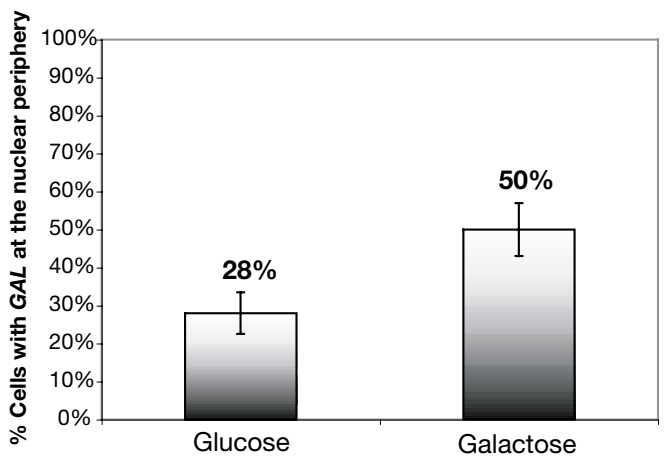

\section{Figure I}

Live-cell imaging of GAL locus intra-nuclear position. (a) A 256-copy lacO array (an 14 kb insert, not drawn to scale) was inserted within the intergenic region between the GAL7 and GALIO genes. This array reports the position of the GAL locus region relative to the nuclear periphery with the co-expression of the GFP-lacl and nucleoporins Nup49 and Nup60 fused to DsRed variant, tdimer2 [23, 24]. (b) The image montage is of one nucleus from a field of view of a single z-plane image sequence. Frames represent $20 \mathrm{~s}$ samples from a 5 min sequence. GAL locus position was scored at the nuclear periphery if the GFP spot mostly overlapped with the red nuclear periphery over the course of an image sequence. (c) GAL locus position was scored not at the periphery if there was no prolonged signal overlap. (d) The percentage of cells that exhibit GAL locus association with the nuclear periphery is enriched in galactose-treated populations versus those grown in glucose. Percentages represent four separate experiments with at least 65 cells scored for each treatment group. Error bars represent the standard deviation across experiments.

It should be noted that in both transcriptionally induced and repressed cultures we observed the same types of GAL locus motion behaviors, but in different proportions. In total, we observed three major types of motion behavior exhibited in cell populations in both glucose and galactose conditions (Figure 2, a-c): loci that are restricted to the nuclear periphery (Figure $2 \mathrm{a}$ ), transiently associated with the nuclear periphery (Figure $2 \mathrm{~b}$ ), or never associated with the periphery (Figure $2 \mathrm{c}$ ).
Using manual visual scoring of GAL loci at the nuclear periphery in live-cell image sequences, we tested whether a key mRNA export factor, Sac3, was involved in the localization of active genes to the nuclear periphery. We constructed a knockout of SAC3 in the lacO-tagged GAL locus haploid strain. The lack of Sac3 prevented the normal galactose-dependent increase in the per cent of cells with the GAL locus at the nuclear periphery (a $22+/-6 \%$ enrichment in SAC3(+) cells, but only a $4+/-1 \%$ enrich- 
a
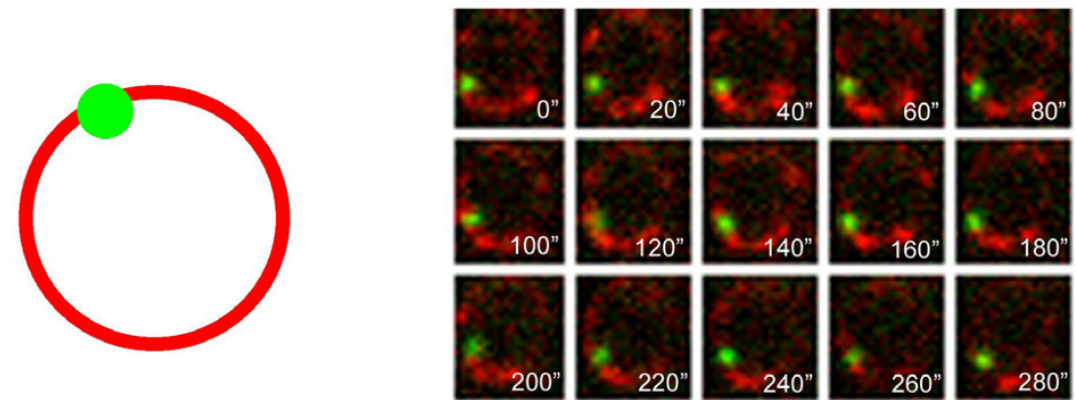

b
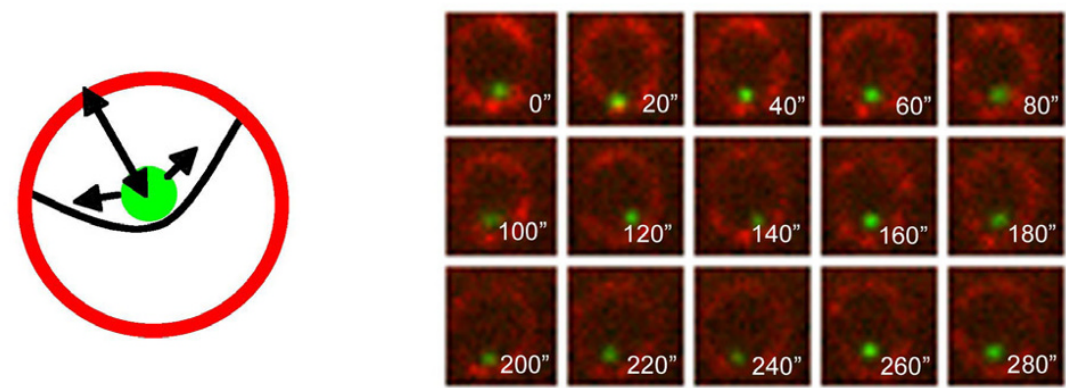

$200 "$

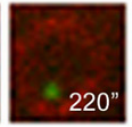

c
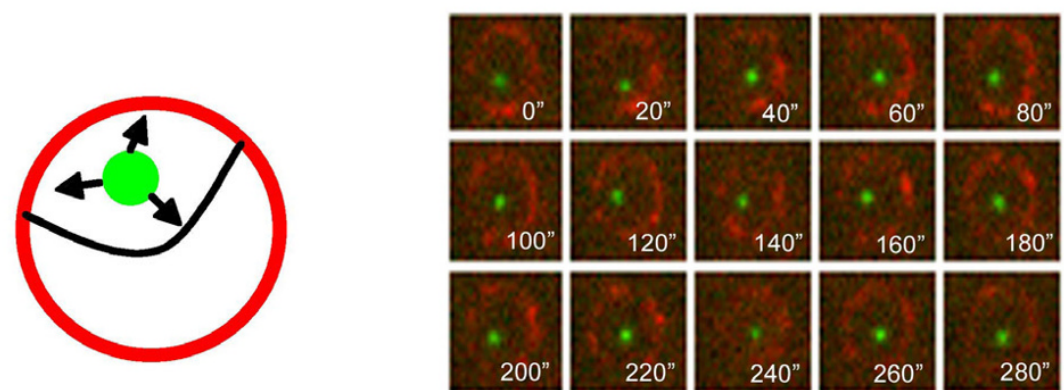

\section{Figure 2}

Three general types of GAL locus motion are observed in yeast populations. (a-c) The image montages represent 20 $\mathrm{s}$ samples of an at least $5 \mathrm{~min}$ image series, where auto-focus was used for the green channel. (a) The GAL locus is restricted to the nuclear periphery, such that the GAL signal visually overlaps with the NPC signal. (b) The GAL locus is not restricted to the nuclear periphery, but exhibits transient associations. (c) The GAL locus exhibits no association with the nuclear periphery during the time course of image-capture. 
ment in sac3 mutant cells), supporting a role for this protein in linking active-genes to the NPC.

\section{GAL locus dynamics in transcriptionally induced and repressed conditions}

To characterize differences in GAL locus motion relative to sub-nuclear location and associated transcriptional activity, we captured single-nucleus image sequences with auto-focus for the GAL locus, similar to prior studies [18] of chromosome dynamics. We first compared our data with that previously established for general yeast chromosome motion $[18,19]$, and performed a mean squared displacement analysis (MSD) to determine differences in spatial constraints of diffusion $[18,19]$. An extension of the open source software ImageJ [25], called SpotTracker [26], was used to align the nuclear periphery signal for each frame to compensate for nuclear motion and subsequently calculate the nuclear center and position of the GAL locus [26]. In order for SpotTracker to accurately calculate the nuclear center, parameter optimization was required for the software to effectively find the nuclear periphery. From these data, the distance, d, between nuclear center and GAL locus was determined for each frame. For free diffusion, the plot of the square of the average change in $d\left(\Delta \mathrm{d}^{2}\right)$ versus particular time intervals $(\Delta \mathrm{t})$ would yield a straight line with a positive slope. Under repressed conditions (in glucose), where GAL loci are largely not at the nuclear periphery, we see by MSD calculation that GAL locus motion is constrained as it yields a curve demonstrating an increasing slope followed by a plateau (Figure 3a) as previously reported for other chromosomal regions $[18,19]$. The plateau seen here is at $\sim 0.05 \mu \mathrm{m}^{2}$, similar to that reported for the centromeres of yeast chromosomes III and IV $[18,19]$. This represents a confinement radius estimated at $\leq 0.3 \mu \mathrm{m}[18,19]$. This is consistent with the chromosomal position of the GAL locus and lacO tag, which are centromere proximal at approximately $37 \mathrm{~kb}$ from the centromere of chromosome II (see Figure 1). Not unexpectedly, we find motion of the GAL locus much more constrained when associated with the nuclear periphery (Figure $3 \mathrm{~b}$ ), which is enriched for upon galactose induction. The corresponding MSD curve is very similar to that reported for telomeres [18], which are known to be highly associated with the NPC and nuclear periphery $[8-10,27,28]$.

\section{Machine visual screening of GAL locus motion}

To further elucidate the mechanisms of active-gene recruitment to the nuclear periphery, we sought an easier way to study gene localization. Current assays for gene localization at the NPC involve chromatin immunoprecipitation (ChIP), fluorescence in situ hybridization (FISH), or manual analysis of live-cell imaging, all of which are time consuming and/or technically demanding. Here we examine the use of real-time chromosome motion in live cells, coupled with machine visual screening as a reporter system for gene localization at the nuclear periphery. Unlike the previously described MSD analysis employed with SpotTracker, machine visual screening should not involve any pre-analysis parameter optimization or geometric segmentation of the visual data, resulting in easier and more accurate analyses.

To demonstrate the application of machine visual screening to the study of chromosome dynamics, we took the same image series analyzed by traditional geometric methods above and applied the machine visual screening software, Visible Discovery [29]. This software generates an aggregate change index (ACI), which is a measure of movement; in this case how spatial organization of fluorescent intensity shifts over time for each frame of the green and red channels. ACI generation does not require parameter optimization or machine-visual segmentation of the GAL locus or the nuclear periphery; all intensity values over the period of the experiment are used (see Methods for a mathematical description of ACI). The calculation of ACI for a given frame involves a comparison with all of the other frames in an image series, so that a frame with a large overall change relative to the rest of the image series will have a larger ACI than other frames.

To compare the motion of the nucleus and GAL locus described by ACI, a diffusion index was generated for each time point (in each channel) $t$; the diffusion index is the root mean square of ACI, for the ACI's of all frames up to and including time $t$. In this fashion, we calculate the way ACI changes over time (see Methods). The diffusion indices of the green channel are then compared via a Pearson correlation to the red channel. If the GAL locus were physically connected to the structural framework of the nucleus, such as the nuclear periphery, or both undergoing similar motion patterns, we would find a positive correlation between the two. On the other hand, lack of correlation between the motion pattern of the GAL locus and the nuclear periphery would strongly suggest the two are not physically connected. We found that indeed, when the GAL locus was scored at the nuclear periphery for cells grown in galactose (the same cells used in the MSD analysis), the correlation between the two channels shows a consistent positive correlation (above $\sim 0.5$ ). In contrast, the correlation of the channels from glucose-grown cells for which the GAL locus was not scored at the periphery ranged from around 0 to strongly negative (Figure $3 \mathrm{c}$ and 3d).

\section{Discussion}

The transcriptional regulation of genes has been linked to their sub-nuclear positioning, particularly relative to the periphery of the nucleus. The nuclear periphery has been traditionally characterized as a zone of repression. How- 
a

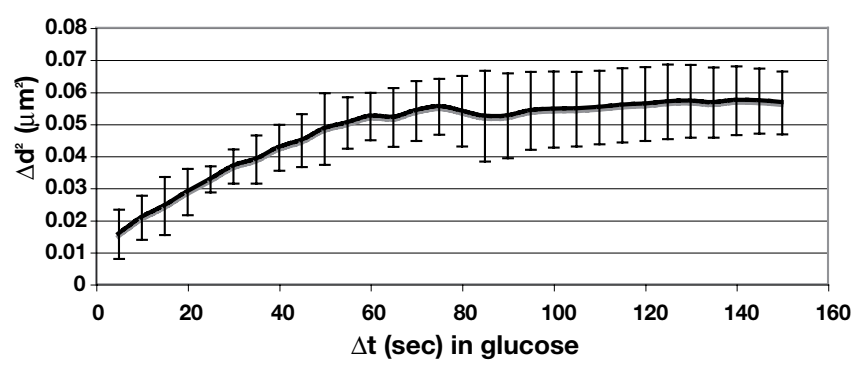

b

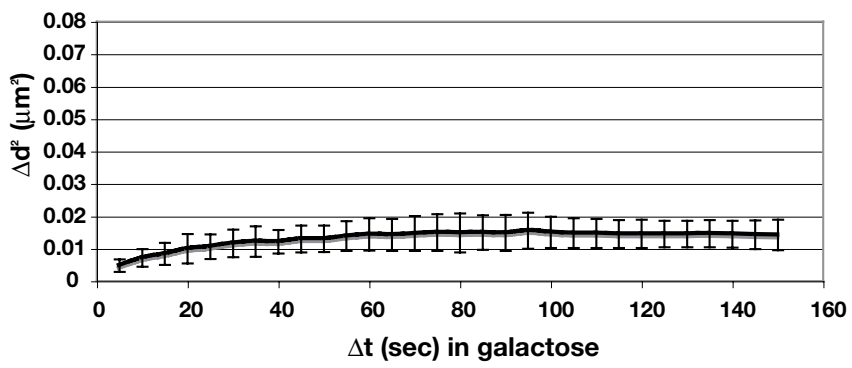

c

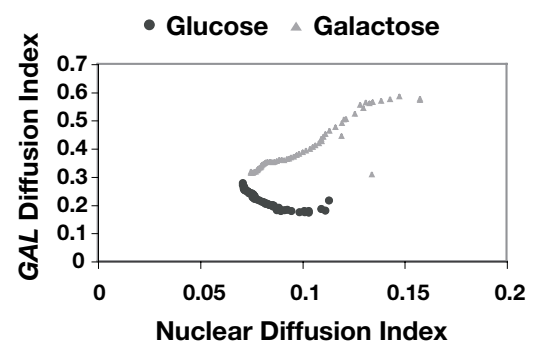

d

\begin{tabular}{lcc}
\multicolumn{3}{c}{ Correlations of Nuclear and GAL Motion } \\
& Glucose & Galactose \\
Cell 1 & -0.0156 & 0.8941 \\
Cell 2 & 0.0818 & 0.7442 \\
Cell 3 & -0.7323 & 0.4858 \\
Cell 4 & -0.4924 & 0.5866 \\
Cell 5 & 0.0892 & 0.7030
\end{tabular}

Figure 3

GAL locus motion analysis. (a, b) Mean square displacement (MSD) analysis was performed as described [I8-20]. The displacement in $\mu \mathrm{m}$ of the GAL locus was estimated as the change in distance $(\Delta d)$ over a period of time $(\Delta t)$ in s. The square of the displacement $\left(\Delta d^{2}\right)$ was calculated as a function of time interval such that $\Delta d^{2}=\{d(t)-d(t+\Delta t)\}^{2}$, for $\Delta t$ that ranged between 5 to $150 \mathrm{~s}$. The graphs are the average of all $\Delta \mathrm{d}^{2}$ for each $\Delta t$ from five single-nucleus image sequences of at least 5 min in length versus $\Delta t$. Error bars represent the standard deviation of $\Delta d^{2}$ for each $\Delta t$ across the five experiments. (a) MSD analysis for GAL loci not scored at the nuclear periphery in glucose conditions. (b) MSD analysis for GAL loci scored at the nuclear periphery in galactose conditions. (c) The graphs represent examples of the plotted correlation between the nuclear and GAL diffusion indices (from Visible Discovery software analysis) over all time points of an image sequence for cells grown in galactose (with GAL at the nuclear periphery), ( $)$ or glucose (with GAL not at the nuclear periphery); $(\bullet)$. Each point represents the diffusion indices (see text) of the nucleus and GAL locus at a specific time $t$. (d) The listed values are the Pearson correlations of nuclear and GAL diffusion indices for five independent nuclei of cells grown in glucose and galactose conditions (same five cells in each condition as analyzed by MSD analysis in (a) and (b)).

ever, recent work from our laboratory and others showing the presence of active genes [14-17] has established that the regulatory milieu at the nuclear periphery is more intricate than first thought.

There are several prevailing ideas as to how and why activated genes relocate to the nuclear periphery. Work by Menon et al [17] suggests that NPC components themselves are involved in transcriptional activation, and that genes are repositioned in the nucleus in order to be brought into a concentrated region of transcription factors for activation. Our laboratory has recently shown the opposite, that the interaction of the NPC component Mlp1 with genes induced by the yeast mating pheromone alpha factor is dependent upon RNA, suggesting that gene association with the nuclear periphery occurs after transcription has begun [15]. Work by Brickner and Walter [16] detailing recruitment of the INO1 locus to the nuclear periphery upon induction, demonstrated that artificial recruitment of the INO1 gene to the nuclear membrane altered the regulation of the gene, but did not directly result in its activation. To better clarify the mech- 
anism behind active-gene recruitment to the NPC in yeast, we assessed the transcription-associated chromosome dynamics in live-cells, specifically of the inducible $G A L$ gene locus.

We confirmed that lacO/GFP-lacI tagging of the GAL locus was representative of GAL localization. Previously, via FISH and NPC-immunofluorescence (FISH/IF), we demonstrated that the GAL locus was at the nuclear periphery with a slightly greater enrichment than observed here. However, the image sequence element of this work may result in differences, as several GAL loci scored at the nuclear periphery in the FISH/IF technique likely represent transient associations at the periphery. Furthermore, the percentages reported here are similar to those observed in the fixed-image, lacO/GFP-lacI reporting of the INO1 intra-nuclear position in response to transcriptional induction [16].

The lacO-tagging technique has been previously used to track the motion of several different chromosome regions in yeast $[18,19]$. In these prior studies, the motion of chromosomes is defined as constrained diffusion, and our data is consistent with this classification. One study, using a similar 2-dimensional tracking, showed that telomeric regions exhibited the greatest motion constraint throughout interphase, while centromeres had an intermediate constraint, and ARS regions had the least constraint [18]. Furthermore, S-phase and the process of replication resulted in the specific increase in constraint of ARSregion motion [18].

Here we have shown a similar "constraining" phenomenon for the GAL locus that is dependent upon transcription-activating conditions, and associated with the specific position of the locus at the nuclear periphery. The GAL genes are located near $(\sim 37 \mathrm{~kb})$ the centromere of yeast chromosome II, and, as expected, exhibit a similar degree of motion constraint when not restricted to the NPC as that reported for the centromeres of yeast chromosomes III and IV $[18,19]$. When found positioned at the nuclear rim, GAL locus motion was highly constrained, comparable to previous observations for telomeric regions [18] and suggesting a physical anchorage to the NPC [14]. Such restriction in movement is in agreement with data showing peripheral localization correlates with increased motion constraint in human cells [30]. This notion is further supported by analysis with the Visible Discovery software [29], in which the motion of the GAL locus is positively correlated with the movement of the nucleus when induced and at the periphery.

Interestingly, when not restricted to the nuclear rim, the GAL locus often exhibited transient overlaps with the nuclear periphery. This is significant as these transient interactions may bring specific factors (nuclear pore and gene-associated) into proximity. The result of a successful interaction would be GAL locus constraint at the nuclear membrane, which is what we observe upon GAL induction. That we still see a fraction of cells with GAL loci not at the periphery in galactose-induced populations may reflect the stochasticity of gene expression in a population of cells (for review see ref. [31]), coupled with a certain probability of gene-NPC interaction. The probability of interaction would be a function of both the chance of a transient interaction of the GAL gene with the NPC resulting from a chromosome's constrained random walk and the on-rate of the interacting factors.

\section{A model for dynamic genomic compartmentalization involving transcription and $m R N A$ export}

If our model suggests there is an increased affinity of the gene for the nuclear periphery upon transcription-activating conditions, what might be responsible for this increased affinity? Previous work from our lab has suggested that RNA is a mediating factor of active-gene and NPC component connectivity [15]. This suggests that a number of mRNA-binding proteins and export factors, which have an affinity for the NPC and associated components, could bridge the interaction between a transcribing gene and the NPC. In these studies, we showed that at least one key factor involved in transcriptional enrichment of loci at the nuclear periphery is the mRNA export protein Sac3. Given the vast array of interactors documented for Sac3 [32-35], there are a number of possibilities for how Sac3 may link activated genes to the nuclear periphery. Sac3 is localized at the NPC [32-34,36], and interacts with transport factors [32,34] and a component of the transcription-activating SAGA complex [37], Sus1 [35]. Interestingly, the localization of Sus 1 at the nuclear periphery is disrupted in the absence of Sac3 [35], perhaps indicating a lack of SAGA-dependent genes, such as the GAL genes [38-42], at the nuclear rim.

Based on these data, we propose a working model for active-gene recruitment to the NPC (Figure 4). As transcription occurs, the appropriate mRNA binding proteins are recruited and deposited on the nascent transcript. It is at this point we propose the cognate gene region has acquired an increased affinity towards the NPC, via Sac3. The active-gene region, which now hosts many Sac3-interacting proteins, is now primed for capture at the NPC.

\section{Chromosome dynamics as a potential phenotypic screen}

Upon characterizing the motion of the GAL loci by the previously employed segmented geometric particle tracking methods, we desired to harness the differences in motion observed between NPC localized GAL and subnuclear GAL to aid in the further phenotypic characterization of tethering of active genes to the NPC. To this end, 
1.
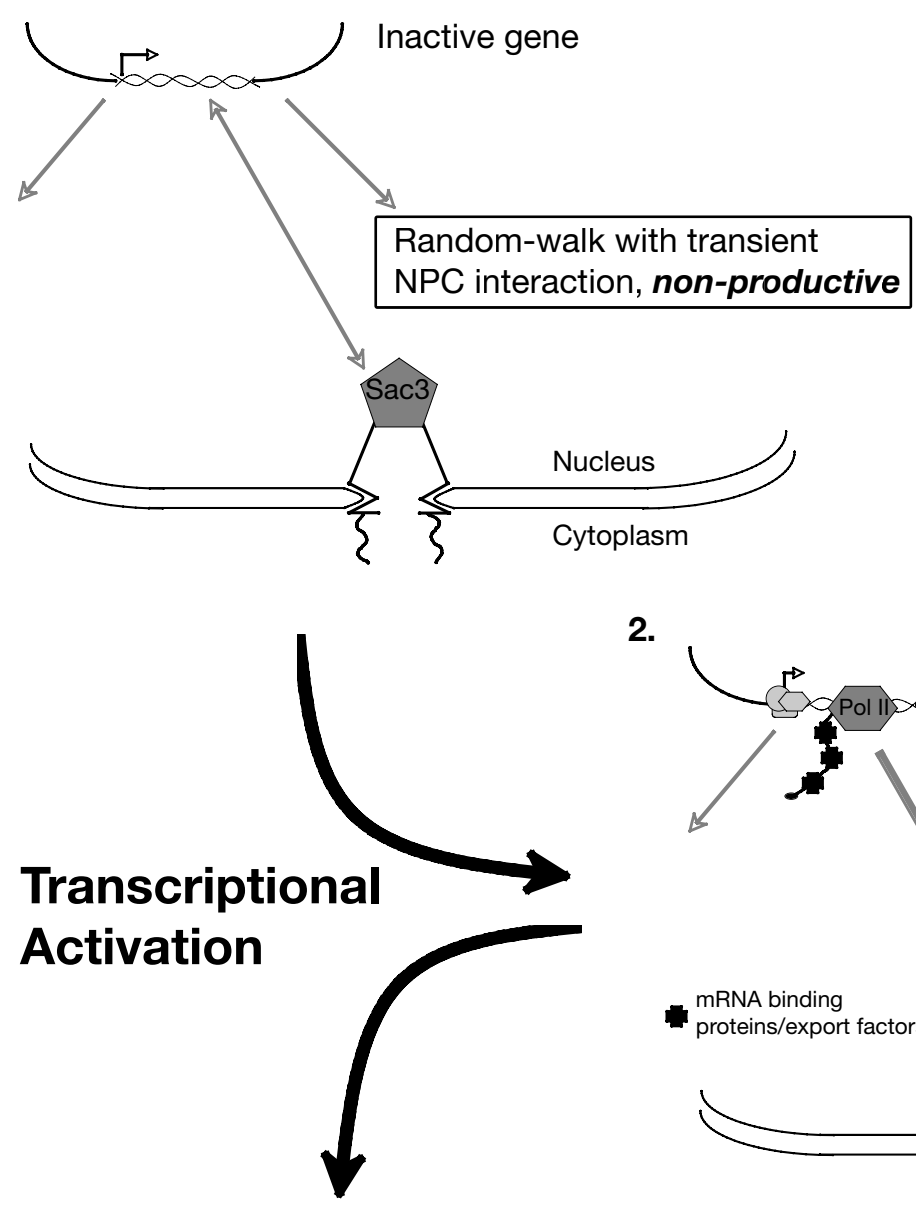

2.

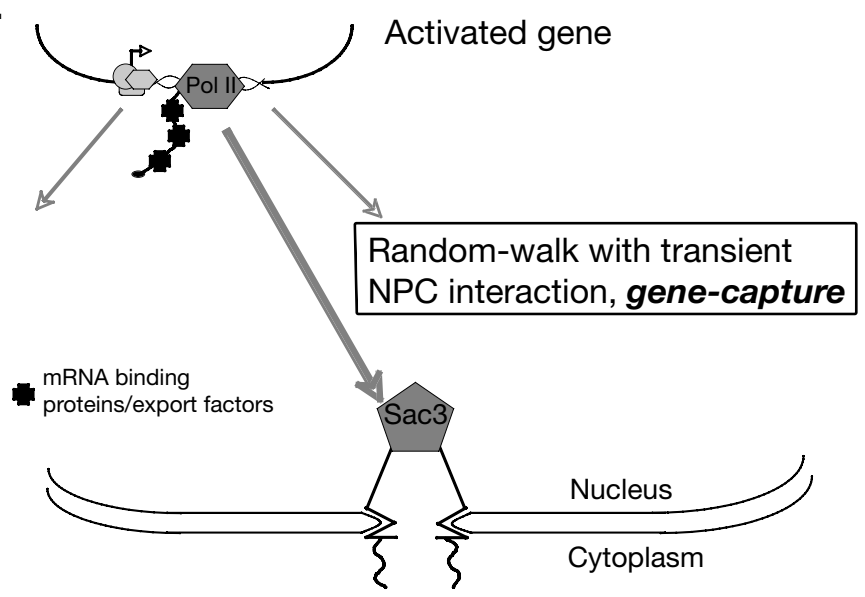

3.

Gene constrained at the NPC via active transcription and Sac3

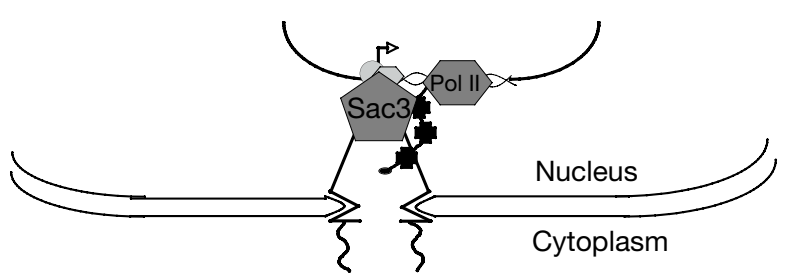

Figure 4

A model for active-gene recruitment to the NPC. When repressed and inactive, an inducible gene exhibits nucleoplasmic diffusion with occasional transient sampling of the nuclear periphery and NPC. Upon transcriptional induction, the transcription machinery is recruited to the gene promoter and transcription is initiated. Appropriate RNA-binding proteins are recruited and deposited upon the nascent mRNA. The activated gene locus now contains many factors with an affinity for NPC-associated Sac3. Once the active locus samples the nuclear periphery after nascent transcription has begun, the gene is captured and constrained at the NPC via Sac3. 
we tested the Visible Discovery machine visual screening analysis software, which is more automated than geometric particle-tracking methods. We demonstrated that, when corralled at the nuclear periphery, the motion of the GAL locus was correlated with that of the nucleus, as inferred by automated measurements of movement indices. This not only reinforces the model that genes become physically associated with the NPC at the nuclear envelope, but also demonstrates that movement is a phenotype that can be easily queried.

The geometric particle tracking methods (such as the MSD analysis) require various estimations and assumptions about the data for analysis and currently requires extensive tweaking and manipulation of the data for the software to "find" the nuclear periphery and spot center. The Visible Discovery analysis platform frees us from these restraints and tedious preparations, and is much more amenable to adaptation for high-throughput analysis. In this case, a visual field of live cells with a tagged GAL locus and nuclear periphery could be monitored over a time course and each nucleus analyzed. (To eliminate the need for auto-focus on one cell at a time, a z-stack of the field could be captured and projected as a 2-dimensional frame for each time point.) A percentage of cells in which the GAL locus motion is correlated with nuclear motion could then be quickly calculated. This motion correlation could, conceptually, also be used for screens of gene associations with different nuclear compartments. For our purposes, to make sure a gene showing correlated motion with the nucleus was actually associated with the nuclear periphery, a simple automated check of signal localization could be made.

An experimental screen would involve the perturbation of the system via mutants or chemical treatments in which this percentage of cells would be assessed for change. In such a way, the molecular details of the mechanism linking active genes with the NPC could be elucidated. Furthermore, the use of motion as an easily screened phenotype could be employed for the studies of many other systems. As we have defined a means of easily monitoring the connectivity of a gene with components of the nuclear periphery, any process involving a gene-nuclear rim association, such as yeast telomere positioning, could be monitored in this fashion.

\section{Conclusion}

This work demonstrates the transcription-associated dynamics of the inducible $G A L$ locus in yeast populations, and a method for machine-screening chromosome localization. Observations of GAL locus movement in populations of transcriptionally induced and repressed conditions lead us to a proposed model for recruitment of an activated gene to the NPC: recruitment results from the random sampling of the nuclear periphery by a gene locus undergoing diffusion that is "captured" upon activation by a mechanism dependent upon Sac3 and nascent mRNA (Figure 4). The machine visual screening analysis, coupled with a high throughput study design, will allow for the easier screening of mutants or chemical treatments on active gene recruitment to the NPC in order to further dissect the molecular mechanism of the process. Furthermore, such a motion phenotype-based screen could be applied to the study of other functions involving alterations in chromosome motion and/or nuclear sub-compartmentalization.

\section{Methods}

\section{Yeast strains, plasmids, and growth conditions}

To integrate the lacO array at the GAL locus between GAL7 and GAL10, a $1 \mathrm{~kb}$ region containing the GAL7/10 intergenic region was first PCR amplified from a chromosome preparation of haploid strain PSY2156 (MATa, ade2-1, trp1-1, can1-100, leu2-3, 112, his3-11, 15, ura3) [14,15,43] using the following primer sequences: 5'-GTGAGCTCCTATTGGTAACCATACAG-3' and 5'-GTGGTACCGAGGCAAAGCAATTTAAG-3', such that the ends of the PCR product contained SacI and KpnI sites. The PCR fragment was cut and ligated into the PAFS59 vector containing the lacO array $[19,21,22]$, resulting in plasmid pPS3112. pPS3112 was linearized for integration using NcoI. The gene encoding GFP-lacI was integrated at HIS3 by cutting plasmid pAFS135 with NheI $[19,21,22]$. Fluorescently tagged nucleoporins, Nup49 and Nup60, were generated via $\mathrm{C}$-terminal fusions of the native genes with a yeastoptimized DsRed variant, tdimer2, using PCR-amplified cassettes $[23,24]$. The final strain was PSY3364 (MATa, ade2-1, trp1-1, can1-100, NUP49-tdimer2,NUP60tdimer2, lacOx256, GFP-lacI). For knockouts of SAC3 in PSY3364 (creating PSY3368), a PCR-amplified knockout cassette was used, encoding the nourseothricin resistance marker [44]. All integrations were verified by PCR. All growth is at $30^{\circ} \mathrm{C}$ unless noted otherwise.

For the comparison of galactose induction and glucose repression, cells were first grown from a streaked plate overnight in a $10 \mathrm{~mL}$ culture in YP media containing 2\% raffinose. As the yeast strains used were ade2-, $20 \mu \mathrm{g} / \mathrm{mL}$ of adenine (Sigma-Aldrich, Inc, St. Louis, MO) was added. Cultures of mid-log phase density were separated in half the next day, both halves spun down, and one half re-suspended in $10 \mathrm{~mL}$ of YP media containing $2 \%$ galactose and the other half re-suspended in $10 \mathrm{~mL}$ of YP media containing $2 \%$ glucose. Re-suspended cultures were grown for 3-hours.

\section{Sample preparation and image capture}

Samples were prepared on plain microslides that were coated with $2 \%$ YP-agarose His- medium with $2 \%$ glucose 
or galactose. For slide coating, $\sim 6 \mu \mathrm{L}$ of a heated $2 \%$ agarose solution was applied to a slide and a coverslip placed on top. The agarose was allowed to harden for at least 40 minutes and the coverslip was slid off so as to preserve a thin coating on the slide. Cells were harvested by centrifugation, washed once with water, and resuspended in 100 $\mu \mathrm{L}$ of minimal media with $2 \%$ glucose or $2 \%$ galactose. About $4 \mu \mathrm{L}$ of cell suspension was placed on the agarose coated slide and a $22 \times 22 \mathrm{~mm}$, no. 11/2, coverslip applied. Coverslips were sealed with Valap (1:1:1 petroleum jelly, lanolin, and paraffin). Cells were imaged using a Nikon TE2000U inverted microscope with PerkinElmer ultraview spinning disk confocal and a $100 \times$ oil objective. For collecting a sequence of a single z-plane field of cells, cells were imaged at $23^{\circ} \mathrm{C}$ for 5 min with capture every 5 $\mathrm{s}$. Exposure for the green channel (488 $\mathrm{nm}$ excitation) was $300 \mathrm{~ms}$, and the red channel (568 nm excitation) was 700 ms using a binning of 2 , such that 1 pixel $=0.13 \mu \mathrm{m}$. Sequences of single cells for tracking were captured in a similar fashion for at least $5 \mathrm{~min}$, except auto-focus on the GAL locus was used in Metamorph 6.3r2 (Molecular Devices, Chicago, IL).

\section{Data analysis}

For visually scoring GAL loci position in an image series, loci visible for most of the 5 min time course were counted at the periphery if locus-overlap with the NPC signal appeared continuous, others were counted as not at the periphery. Cells with two loci were not scored. About ten different fields of view were captured as an image sequence for each treatment or growth condition, at least in triplicate. Cells with more than two visible loci, and those with deformed nuclear envelopes, were not counted.

We used both an established as well as a novel method to determine the extent to which the motion pattern of the $G A L$ locus was constrained or freely diffusive. Identical image sequences of individual nuclei (five for each condition, glucose and galactose) were used for all analysis.

\section{SpotTracker}

Single particle tracking methods using mean square displacement (MSD) have been previously established for quantifying movement characteristics. SpotTracker is a plug-in for the software ImageJ [25]. SpotTracker algorithms and parameter sets have been previously detailed [26]. SpotTracker effectively assigns $x, y$ coordinates of the nuclear center and GAL locus spot for each frame. For each image sequence, calculated GAL locus and nuclear center coordinates were input into Microsoft Excel to calculate the distance between them $(d)$ and the $\Delta d^{2}$ as a function of the time interval $(\Delta t)$ such that $\Delta d^{2}=\{\mathrm{d}(t)-\mathrm{d}(t+\Delta t)\}^{2}$. A set of user parameters for intensity thresholds, fitting parameters, and confidence thresholds, dependent upon the image sequence, were optimally set for this analysis. These values and settings were set empirically based upon the raw image data being analyzed.

\section{Machine visual screening}

Visible Discovery (Reify Corporation, Cambridge, MA) [29] is a proprietary machine visual screening software platform that we used here to calculate two parameters, the diffusion index (Ds), and the Aggregate Change Index (ACI). These parameters are defined for an ordered series of $n$ frames defined by an intensity function $I_{x, y, t}$ where $x$ and $y$ are integers defining the coordinates of a given pixel in a particular frame, and $t$ is an integer corresponding to the time of capture. Frames are captured at equally spaced intervals. The equations below are based on the more general equations given in [45].

First, a similarity function $S M$ is calculated for all pairs of frames taken at times $i$ and $j(i \neq j)$ :

$$
S M_{i, j}=\frac{\left[N \sum \sum I_{x, y, i} I_{x, y, j}-\left(\sum \sum I_{x, y, i}\right)\left(\sum \sum I_{x, y, j}\right)\right]^{2}}{\left\{\left[N \sum \sum I_{x, y, i}{ }^{2}-\left(\sum \sum I_{x, y, i}\right)^{2}\right]\left[N \sum \sum I_{x, y, j}{ }^{2}-\left(\sum \sum I_{x, y, j}\right)^{2}\right]\right\}}
$$

Where $N=w h$, and each double summation is calculated over the width $w$ and height $h$ of the frame; i.e., $\sum_{x=0}^{w} \sum_{y=0}^{h}$, and is calculated for all pairs of $i$ and $j$ where $i \neq j$. The similarity function is commutative, such that $S M_{i, j}=S M_{j, i}$. Furthermore, $S M_{i, j}=1$ if $\mathrm{i}==\mathrm{j}$.

A normalized $S M_{i, j}$ function, called $P_{i, j}$ is then calculated for each $S M_{i, j}$ of an image series.

$$
P_{i, j}=\frac{S M_{i, j}}{\sum_{k=0}^{n} S M_{i, k}}
$$

The Aggregate Change Index for a given frame $i$ is calculated as follows:

$$
A C I_{i}=\sum_{j=0}^{n} P_{i, j} \log _{2}\left(P_{i, j}\right) / \log _{2}(n)
$$

The Diffusion Index Ds is calculated based on ACI as:

$D s_{t}=\sqrt{\frac{\left(\sum_{i=0}^{t} A C I_{i}^{2}\right)}{n}}$

In such a way, Visible Discovery computes a diffusion index from an ACI for the independent image sequences (green 
channel and red channel) of both the GAL locus and nuclear periphery. No pre-processing or parameter tuning was applied. Software execution time for a single movie was less than a $100 \mathrm{~ms}$ on a $1 \mathrm{Ghz}$ Macintosh G4 running OS X.

ACI is a self-similarity temporal index (see [46] for characterization of Brownian motion as a self-similar process) and is an estimate of the temporal autocorrelation function. A diffusion index at any time point $t$ is the root mean square of $\mathrm{ACI}$ values from and including the first time point up to $t$. Without compensation for drifts, ACI assumes that instantaneous displacements are within a small envelope of the autocorrelation function.

Random diffusion or transport can be characterized either from correlation of velocities, or correlation of positions [47]. MSD analysis calculates the average of displacements over fixed interval times, i.e. a velocity correlation function. ACI is a position correlation function, i.e. variations of correlation of a process calculated spatially over time. ACI analysis does not attempt to model the underlying process. Rather, ACI computes a trend of how the underlying process changes over time. Computing ACI, requires comparing every image in the sequence to every other image. Image sequences were 2 dimensional arrays, or regions within, of pixel values. We used normalized cross-correlation to measure the similarity function between two images.

\section{Authors' contributions}

DAD carried out all the biological aspects of the study, performed the geometric MSD analysis, participated in the experimental design and conceptualization of the project, and drafted the manuscript. AMG supplied software expertise and design in performing all the machine visual screening analysis and its description in the manuscript. PAS participated in the design and coordination of the study and helped in the process of developing the manuscript. All authors read and approved the final manuscript.

\section{Acknowledgements}

We thank A. Murray for supplying the lac operator system plasmids, K. Thorn and R. Tsien for the tdimer2-tagging vectors, L. Petrak and J. Waters with the Nikon Imaging Center at Harvard Medical School as well as K. Auld and C. Ajo-Franklin for technical assistance, and C. Brown, M. McLaughlin-Drubin, and J. Way for manuscript comments. This work was supported by an NIH Institutional NRSA fellowship to D.A.D. and grants from the NIH to P.A.S.

\section{References}

I. Oliver B, Misteli T: A non-random walk through the genome. Genome Biol 2005, 6(4):214.

2. Cohen BA, Mitra RD, Hughes JD, Church GM: A computational analysis of whole-genome expression data reveals chromosomal domains of gene expression. Nat Genet 2000, 26(2): $183-186$.
3. Gilbert N, Boyle S, Fiegler H, Woodfine K, Carter NP, Bickmore WA: Chromatin architecture of the human genome: gene-rich domains are enriched in open chromatin fibers. Cell 2004, I I 8(5):555-566.

4. Cockell M, Gasser SM: Nuclear compartments and gene regulation. Curr Opin Genet Dev 1999, 9(2): 199-205.

5. Gilbert N, Gilchrist S, Bickmore WA: Chromatin organization in the mammalian nucleus. Int Rev Cytol 2005, 242:283-336.

6. Misteli T: Spatial positioning; a new dimension in genome function. Cell 2004, I I 9(2): I53-I56.

7. Zink D, Amaral MD, Englmann A, Lang S, Clarke LA, Rudolph C, Alt F, Luther K, Braz C, Sadoni N, et al.: Transcription-dependent spatial arrangements of CFTR and adjacent genes in human cell nuclei. J Cell Biol 2004, I 66(6):8I5-825.

8. Feuerbach $\mathrm{F}$, Galy $\mathrm{V}$, Trelles-Sticken E, Fromont-Racine M, Jacquier A, Gilson E, Olivo-Marin JC, Scherthan H, Nehrbass U: Nuclear architecture and spatial positioning help establish transcriptional states of telomeres in yeast. Nat Cell Biol 2002, 4(3):2I 4-22I.

9. Galy V, Olivo-Marin JC, Scherthan H, Doye V, Rascalou N, Nehrbass $\mathrm{U}$ : Nuclear pore complexes in the organization of silent telomeric chromatin. Nature 2000, 403(6765): 108-I I2.

10. Gotta M, Laroche T, Formenton A, Maillet L, Scherthan H, Gasser SM: The clustering of telomeres and colocalization with Rap I, Sir3, and Sir4 proteins in wild-type Saccharomyces cerevisiae. J Cell Biol I 996, I34(6): 1349-1363.

II. Laroche T, Martin SG, Gotta M, Gorham HC, Pryde FE, Louis EJ, Gasser SM: Mutation of yeast Ku genes disrupts the subnuclear organization of telomeres. Curr Biol 1998, 8(II):653-656.

12. Andrulis ED, Neiman AM, Zappulla DC, Sternglanz R: Perinuclear localization of chromatin facilitates transcriptional silencing. Nature 1998, 394(6693):592-595.

13. Ishii K, Arib G, Lin C, Van Houwe G, Laemmli UK: Chromatin boundaries in budding yeast: the nuclear pore connection. Cell 2002, 109(5):55I-562.

14. Casolari JM, Brown CR, Komili S, West J, Hieronymus H, Silver PA: Genome-wide localization of the nuclear transport machinery couples transcriptional status and nuclear organization. Cell 2004, I I 7(4):427-439.

15. Casolari JM, Brown CR, Drubin DA, Rando OJ, Silver PA: Developmentally induced changes in transcriptional program alter spatial organization across chromosomes. Genes Dev 2005, 19(10): I 188-1198.

16. Brickner $\mathrm{JH}$, Walter P: Gene Recruitment of the Activated INOI Locus to the Nuclear Membrane. PLOS Biol 2004, 2(II):E342.

17. Menon BB, Sarma NJ, Pasula S, Deminoff SJ, Willis KA, Barbara KE, Andrews B, Santangelo GM: Reverse recruitment: The Nup84 nuclear pore subcomplex mediates RapI/GcrI/Gcr2 transcriptional activation. Proc Natl Acad Sci U S A 2005.

18. Heun P, Laroche T, Shimada K, Furrer P, Gasser SM: Chromosome dynamics in the yeast interphase nucleus. Science 200I, 294(5549):218I-2I86.

19. Marshall WF, Straight A, Marko JF, Swedlow J, Dernburg A, Belmont A, Murray AW, Agard DA, Sedat JW: Interphase chromosomes undergo constrained diffusional motion in living cells. Curr Biol 1997, 7( I 2):930-939.

20. Vazquez J, Belmont AS, Sedat JW: Multiple regimes of constrained chromosome motion are regulated in the interphase Drosophila nucleus. Curr Biol 200 I, I I ( I6): I 227-I 239.

21. Robinett CC, Straight A, Li G, Willhelm C, Sudlow G, Murray A, Belmont AS: In vivo localization of DNA sequences and visualization of large-scale chromatin organization using lac operator/repressor recognition. J Cell Biol 1996, I35(6 Pt 2): $1685-1700$

22. Straight AF, Belmont AS, Robinett CC, Murray AW: GFP tagging of budding yeast chromosomes reveals that protein-protein interactions can mediate sister chromatid cohesion. Curr Biol 1996, 6( (12): 1599-1608

23. Campbell RE, Tour O, Palmer AE, Steinbach PA, Baird GS, Zacharias DA, Tsien RY: A monomeric red fluorescent protein. Proc Natl Acad Sci U S A 2002, 99(12):7877-7882.

24. Sheff MA, Thorn KS: Optimized cassettes for fluorescent protein tagging in Saccharomyces cerevisiae. Yeast 2004, 2I(8):661-670.

25. Image], U. S. National Institutes of Health [http:// rsb.info.nih.gov/ij/] 
26. Sage D, Hediger F, Gasser SM, Unser M: Automatic Tracking of Particles in Dynamic Fluorescence Microscopy. Proceedings of the Third International Symposium on Image and Signal Processing and Analysis (ISPA'03) 2004:I.582-I.586.

27. Tham WH, Wyithe JS, Ko Ferrigno P, Silver PA, Zakian VA: Localization of yeast telomeres to the nuclear periphery is separable from transcriptional repression and telomere stability functions. Mol Cell 200I, 8(I):189-199.

28. Heun P, Laroche T, Raghuraman MK, Gasser SM: The positioning and dynamics of origins of replication in the budding yeast nucleus. J Cell Biol 200I, I 52(2):385-400

29. Hack AA, Ahouse J, Roberts PG, Garakani AM: New methods for automated phenotyping of complex cellular behaviors. Engineering in Medicine and Biology Society, 2004 EMBC 2004 Conference Proceedings 2004, 2:5 I24-5I 26.

30. Chubb JR, Boyle S, Perry P, Bickmore WA: Chromatin motion is constrained by association with nuclear compartments in human cells. Curr Biol 2002, I 2(6):439-445.

31. Kaern M, Elston TC, Blake WJ, Collins J]: Stochasticity in gene expression: from theories to phenotypes. Nat Rev Genet 2005 , 6(6):45I-464.

32. Fischer T, Strasser K, Racz A, Rodriguez-Navarro S, Oppizzi M, Ihrig $P$, Lechner ], Hurt $E$ : The mRNA export machinery requires the novel Sac3p-ThpIp complex to dock at the nucleoplasmic entrance of the nuclear pores. Embo J 2002, 21 (2I):5843-5852.

33. Jones AL, Quimby BB, Hood JK, Ferrigno P, Keshava PH, Silver PA Corbett AH: SAC3 may link nuclear protein export to cell cycle progression. Proc Natl Acad Sci U S A 2000, 97(7):3224-3229.

34. Lei EP, Stern CA, Fahrenkrog B, Krebber H, Moy TI, Aebi U, Silver PA: Sac3 is an mRNA export factor that localizes to cytoplasmic fibrils of nuclear pore complex. Mol Biol Cell 2003, 14(3):836-847.

35. Rodriguez-Navarro S, Fischer T, Luo MJ, Antunez $\mathrm{O}$, Brettschneider S, Lechner J, Perez-Ortin JE, Reed R, Hurt E: Sus I, a functional component of the SAGA histone acetylase complex and the nuclear pore-associated mRNA export machinery. Cell 2004, I I 6(I):75-86.

36. Huh WK, Falvo JV, Gerke LC, Carroll AS, Howson RW, Weissman JS, O'Shea EK: Global analysis of protein localization in budding yeast. Nature 2003, 425(6959):686-69l.

37. Timmers HT, Tora L: SAGA unveiled. Trends Biochem Sci 2005 , 30(I):7-10.

38. Bhaumik SR, Green MR: SAGA is an essential in vivo target of the yeast acidic activator Gal4p. Genes Dev 200I, I 5(1 5): 1935-1945.

39. Dudley AM, Rougeulle C, Winston F: The Spt components of SAGA facilitate TBP binding to a promoter at a post-activator-binding step in vivo. Genes Dev 1999, 13(22):2940-2945.

40. Roberts SM, Winston F: Essential functional interactions of SAGA, a Saccharomyces cerevisiae complex of Spt, Ada, and Gcn5 proteins, with the Snf/Swi and Srb/mediator complexes. Genetics 1997, I47(2):45I-465.

41. Sterner DE, Grant PA, Roberts SM, Duggan LJ, Belotserkovskaya R, Pacella LA, Winston F, Workman JL, Berger SL: Functional organization of the yeast SAGA complex: distinct components involved in structural integrity, nucleosome acetylation, and TATA-binding protein interaction. Mol Cell Biol 1999, 19(1):86-98

42. Larschan $E$, Winston F: The S. cerevisiae SAGA complex functions in vivo as a coactivator for transcriptional activation by Gal4. Genes Dev 200I, I5(I 5): 1946-1956.

43. Ren B, Robert F, Wyrick J], Aparicio O, Jennings EG, Simon I, Zeitlinger J, Schreiber J, Hannett N, Kanin E, et al:: Genome-wide location and function of DNA binding proteins. Science 2000 290(5500):2306-2309.

44. Goldstein AL, McCusker JH: Three new dominant drug resistance cassettes for gene disruption in Saccharomyces cerevisiae. Yeast 1999, 15(14):154|-1553.

45. Garakani A, Hack A, Roberts P, Walter S: Method and Apparatus for Acquisition, Compression, and Characterization of Spatiotemporal Signals. 1999. U.S. Patent Application US20030I85450AI

46. Taqqu MS: Fractional Brownian motion and long-range dependence. In Theory and Applications of Long-range Dependence Edited by: Doukhan P, Openheim G, Taqqu MS. Boston: Birkhèauser; 2003:5-38.
47. Qian H, Sheetz MP, Elson EL: Single particle tracking. Analysis of diffusion and flow in two-dimensional systems. Biophys J 1991, 60(4):910-921.

Publish with Biomed Central and every scientist can read your work free of charge

"BioMed Central will be the most significant development for disseminating the results of biomedical research in our lifetime. "

Sir Paul Nurse, Cancer Research UK

Your research papers will be:

- available free of charge to the entire biomedical community

- peer reviewed and published immediately upon acceptance

- cited in PubMed and archived on PubMed Central

- yours - you keep the copyright 